\title{
Programa Médicos pelo Brasil: mérito e equidade
}

\author{
Médicos pelo Brasil Program: merit and equity \\ Programa Médicos pelo Brasil: mérito y equidad \\ Lucas Wollmann $^{1 \oplus}$, Otavio Pereira D’Avila ${ }^{2 \oplus}$, Erno Harzheim ${ }^{3}{ }^{\circledR}$ \\ ${ }^{1}$ Grupo Hospitalar Conceição, Porto Alegre, Rio Grande do Sul, Brasil \\ 2 Universidade Federal de Pelotas (UFPel), Pelotas, Rio Grande do Sul, Brasil. \\ ${ }^{3}$ Universidade Federal do Rio Grande do Sul (UFRGS), Porto Alegre, Rio Grande do Sul, Brasil.
}

\section{Resumo}

O Programa Médicos pelo Brasil (PMPB) foi lançado em 2019 pelo Ministério da Saúde com objetivo de ampliar a oferta de serviços médicos em locais de difícil provimento ou alta vulnerabilidade. As principais mudanças propostas no PMPB são: obrigatoriedade de registro no Conselho Federal de Medicina; alocação de vagas com prioridade para as pequenas e distantes cidades, contratação dos profissionais via CLT e formação qualificada em Medicina de Família e Comunidade (MFC), permitindo a titulação dos médicos após dois anos. Com essas mudanças, espera-se um aprofundamento na interiorização dos profissionais, com possibilidade efetiva de fixação, além da formação em larga escala de MFCs. A formação através da residência também será impulsionada, com incentivo financeiro municipal para esse fim, em consonância com as ações do novo financiamento federal da Atenção Primária à Saúde (APS), o Programa Previne Brasil. O PMPB será executado pela Agência para o Desenvolvimento da Atenção Primária à Saúde (Adaps), um modelo inovador de gestão pública, que trará eficiência ao programa. Com essas características, o PMPB pretende oferecer um solução perene para a oferta de serviços médicos no âmbito da APS do Sistema Único de Saúde.

Palavras-chave: Atenção primária à saúde, estratégia de saúde da família, provisão e distribuição de médicos.

\begin{abstract}
The Médicos pelo Brasil Program (PMPB) was launched in 2019 by the Ministry of Health with the objective to expand the supply of medical services in places of difficult provision or high vulnerability. The main changes proposed in the PMPB are: mandatory registration with the Federal Council of Medicine; allocation of vacancies with priority for small and distant cities, hiring of professionals via national labor legislation and qualified training in Family Medicine (FM), allowing the doctor's speciality certification after two years. With these changes, it is expected to deepen the internalization of professionals, with effective possibility of fixation, in addition to training in scale in FM. Training through residency will also be promoted, with municipal financial incentives for this purpose, aligned with the actions of the new federal financing of Primary Health Care (PHC), the Previne Brasil Program. The PMPB will be executed by the Agency for the Development of Primary Health Care (Adaps), an innovative management model that will bring efficiency to the program. With these characteristics, the PMPB intends to offer a solution for the provision of medical services within the PHC of the Brazilian Public Health System.
\end{abstract}

Keywords: Primary health care, family health strategy, provision and distribution of doctors.

Como citar: Wollmann L, D'Avila OP, Harzheim E. Programa Médicos pelo Brasil: mérito e equidade. Rev Bras Med Fam Comunidade. 2020;15(42):2346. https://doi.org/10.5712/rbmfc15(42)2346

\section{Autor correspondente:}

Lucas Wollmann

E-mail: lucasw.bm@gmail.com

Fonte de financiamento:

declaram não haver.

Parecer CEP:

não se aplica.

Procedência e revisão por pares:

Não encomendado; revisão por pares externa. Recebido em: 06/01/2020.

Aprovado em: 03/02/2020. 


\section{Resumen}

El Programa de Médicos pelo Brasil (PMPB) fue lanzado en 2019 por el Ministerio de Salud con el bjetivo expandir la oferta de servicios médicos en lugares de difícil provisión o alta vulnerabilidad. Los rincipales cambios propuestos en el PMPB son: registro obligatorio en el Consejo Federal de Medicina; designación de vacantes con prioridad para ciudades pequeñas y distantes, contratación de profesionales a través de las leyes laborales nacionales y capacitación calificada en medicina familiar y comunitaria (MFC), lo que permite la certificación de especialidad después de dos años. Con estos cambios, se espera profundizar la internalización de profesionales, con posibilidad efectiva de fijación, además de capacitación a gran escala de los MFC. La capacitación a través de la residencia también será promovida, con incentivos financieros municipales para este propósito, en línea con las acciones del nuevo financiamiento federal de Atención Primaria de Salud (APS), el Programa Previne Brasil. EI PMPB será ejecutado por la Agencia para el desarrollo de la atención primaria de salud (Adaps), un modelo de gestión innovador. Eso traerá eficiencia al programa. Con estas características, el PMPB pretende ofrecer un solución perenne para la prestación de servicios médicos dentro de la APS del Sistema Único de Salud.

Palabras clave: Atención primaria de salud, estrategia de salud familiar, provisión y distribución de doctores.

O Programa Médicos pelo Brasil (PMPB) foi proposto pelo Governo Federal através da Medida Provisória $n^{\circ} 890$ de 2019, assinada pelo Presidente da República Jair Bolsonaro e pelo Ministro da Saúde Luiz Henrique Mandetta, em 01 de agosto de 2019, sendo aprovada na Câmara dos Deputados com 391 votos favoráveis e 6 contrários e sancionada pelo Presidente através da Lei 13.958 de 18 de dezembro de 20191. O PMPB é a estratégia da Secretaria de Atenção Primária à Saúde (SAPS) para avançar no provimento e fixação de médicos na Estratégia de Saúde da Família (ESF), corrigindo distorções do programa anterior, o Projeto Mais Médicos (PMM).

Uma das principais mudanças propostas pelo PMPB é a obrigatoriedade de registro nos Conselhos Regionais de Medicina (CRM) para o ingresso no programa. Dois motivos levaram a essa decisão: o registro no CRM oferece liberdade profissional aos médicos do programa, encerrando uma estratégia que se valeu da ausência de livre-arbítrio para fixação de profissionais, inclusive com denúncias de trabalho escravo por parte dos médicos ${ }^{2}$. Além disso, acaba com a certificação profissional provisória oferecida pelo Ministério da Saúde. Essa situação extraordinária criou um cenário de conflito de interesses inadmissível, uma vez que colocou sob a mesma competência, responsabilidades que por interesse público devem ficar separadas: a contratação e a certificação profissional. Como efeito colateral, esse arranjo também incentivou uma verdadeira indústria de faculdades de medicina em países fronteiriços ao Brasil, com dezenas de milhares de médicos interessados com a possibilidade de trabalhar no país sem a necessidade do devido processo de revalidação de diploma³.

Outra mudança importante do PMPB, são os novos critérios de alocação das vagas nos municípios. Os principais fatores de dificuldade de atração de médicos no Brasil são são o tamanho de uma cidade e a sua distância de um grande centro urbano ${ }^{4}$. Dessa forma, o PMPB priorizará as pequenas e distantes cidades brasileiras, bem caracterizadas pelo Ministro da Saúde como o "Brasil Profundo". O PMPB utilizará estudo do Instituto Brasileiro de Geografia e Estatística, embasado em estudo da Organização para a Cooperação e Desenvolvimento Econômico (OCDE), para classificar essas cidades ${ }^{5}$. As cidades mais distantes e com menor porte terão prioridade para receber médicos do PMPB, em todas as suas ESF. As equipes em distritos sanitários especiais indígenas e equipes ribeirinhas, fluviais e quilombolas também serão considerados prioritárias. Mas, sabendo das dificuldades que as médias e grandes cidades também apresentam no provimento de médicos nos bairros de maior vulnerabilidade socioeconômica, o 
PMPB também prevê um segundo critério de distribuição de vagas nesses locais. Será utilizado método que possibilitará a identificação das ESF que atendem a maior proporção de pessoas em situação de vulnerabilidade socioeconômica, cruzando as bases de dados de cadastro das ESF com as bases de dados de beneficiários do bolsa-família, benefício de prestação continuada (BPC) e benefícios da previdência social até dois salários mínimos. Nesse caso, o médico será designado para uma ESF específica, garantindo a oferta de serviços para a população mais vulnerável. No PMM, a equipe de alocação do profissional é discricionária do gestor municipal.

As condições de trabalho também exercem influência importante na capacidade de retenção profissional ${ }^{4}$. Para atuar sobre essa situação, em primeiro lugar, o PMPB vai oferecer contratação através da Consolidação das Leis do Trabalho (CLT) aos médicos ingressantes do programa, com plano de cargos e salários, e progressão pré-definida. Em segundo lugar, será oferecido pagamento diferencial para médicos alocados em regiões remotas. Em terceiro lugar, no Termo de Adesão dos municípios, será solicitado o compromisso do gestor municipal com a qualificação da Atenção Primária à Saúde (APS) de sua cidade. Através do novo financiamento da APS, o Previne Brasil, os gestores municipais terão melhores condições de estruturar suas ESF ${ }^{6}$, já que há aumento real no financiamento federal das ESF para 2020. Importante salientar que os médicos do PMPB realizarão uma avaliação da estrutura da APS dos locais onde estão alocados, permitindo o estabelecimento de um canal de comunicação entre médico-município-Ministério da Saúde. Essas mudanças aumentarão a capacidade de fixação efetiva e permanente dos profissionais na APS.

Em relação a remuneração, outro avanço é que os médicos na contratação CLT terão um componente de pagamento por desempenho, incentivando e recompensando a qualidade dos profissionais que melhor atenderem a população. A mensuração desse desempenho acompanhará os mesmos indicadores de saúde e indicadores globais de qualidade da APS por meio de instrumentos validados nacional e internacionalmente do Previne Brasil. Desta forma, gestores, equipes e médicos terão os mesmos desafios de melhora da qualidade da APS.

Além do provimento, o PMPB oferecerá uma formação qualificada em Medicina de Família e Comunidade (MFC), através de curso de especialização de 60hs semanais (40hs assistenciais $+20 \mathrm{hs}$ de atividades teóricas). Os médicos em formação serão supervisionados por um tutor clínico, com quem passarão uma semana a cada dois meses em supervisão contínua. Além disso, receberão uma tutoria acadêmica no componente teórico da formação. Todo o conteúdo do curso está sendo desenvolvimento em parceria com a Sociedade Brasileira de Família e Comunidade (SBMFC), alinhado com seu Currículo Baseado em Competências ${ }^{7}$. Ao final de dois anos, o médico aprovado na especialização estará credenciado a realizar prova de título de especialista em MFC. Espera-se que o aumento rápido do contingente de MFCs no Brasil, além de elevar a qualidade da assistência na APS, produza impacto na demanda social pela especialidade, a partir de seu crescimento e difusão do seu contato no cotidiano da atenção à saúde da população. Estratégia semelhante foi adotada por vários países que, ao reformar seus sistemas de saúde com enfoque na APS, precisaram de um grande número de MFCs.

Os profissionais do PMPB também serão incentivados a utilizar outras ferramentas de suporte clínico e tomada de decisão baseada em evidências. Com a renovação do convênio do Ministério da Saúde com 
o TelessaúdeRS, os médicos na Atenção Primária seguem com a oferta do serviço de teleconsultorias gratuitas, para que possam tirar dúvidas em tempo real sobre diagnóstico e tratamento. Este recurso é ainda mais oportuno em locais distantes dos grandes centros urbanos, ajudando a resolver os problemas de saúde dos usuários de maneira mais rápida. As dúvidas são respondidas por especialistas nas mais diversas áreas. Além das teleconsultorias, os profissionais terão acesso a materiais de apoio, como protocolos de encaminhamento para outros serviços de atendimento especializado e guias de apoio à decisão clínica, baseados nas melhores evidências científicas e adequados à realidade do Brasil. Além disso, estão sendo adquiridos pela SAPS outros materiais de suporte à tomada de decisão clínica na APS, que deverão ser ofertados para todas as equipes que atuam na APS do SUS. Outra oferta inovadora são linhas de cuidado para 22 das mais frequentes condições de saúde, baseadas nas melhores evidências disponíveis, e atribuindo de forma clara as responsabilidades de cada ponto assistencial.

Importante notar uma pequena, mas importante mudança no PMPB: o aumento de $25 \%$ nas horas de trabalho assistencial dos médicos, exigindo 40hs semanais ao invés de $32 \mathrm{hs}$ (exigência do PMM), equivale à contratação adicional de 4,5 mil médicos em relação aos $18 \mathrm{mil}$ do PMM, se fossem mantidas as mesmas $32 \mathrm{hs}$ semanais.

A avaliação dos profissionais é outra novidade importante do PMPB. Os médicos serão acompanhados através de indicadores de produtividade e desempenho técnico relacionados ao cuidado de problemas de saúde prevalentes e importantes (pré-natal, cuidado com doenças crônicas, atividades preventivas). Através de pesquisa direta com usuários, também será avaliada a relação médico-paciente, a qualidade do cuidado recebido e a satisfação com o atendimento. Isso vai permitir o acompanhamento do impacto que cada profissional provocou na equipe a que foi deslocado, dando maior transparência ao programa. No escopo da especialização, serão realizadas avaliações semestrais, além de um trabalho de conclusão que envolve a identificação de um problema de saúde relevante no local onde o médico atua, com uma intervenção que busca mitigar a situação. Também haverá um sistema de avaliação das competências profissionais da especialidade, com a participação do tutor clínico.

Desde seu lançamento, o PMPB foi recebido com análises positivas de diferentes entidades da área da saúde ${ }^{8,9}$, inclusive da SBMFC ${ }^{10}$. Esse apoio pode ser creditado ao fato de o PMPB ter sido elaborado a partir das melhores evidências relacionadas às ações de provimento e fixação de médicos no mundo, mas também pelo intenso diálogo mantido com diversas entidades do setor saúde ao longo da construção do programa.

Por outro lado, críticas ao novo programa também foram feitas ${ }^{11,12}$. Foi argumentado que houve uma redução do escopo das propostas PMPB em relação ao PMM, uma vez que este apresentava ações relacionadas a abertura de faculdades de medicina e programas de residência médica. Mas críticas também podem ser feitas a falha de análise contextual no delineamento da amplitude da política anterior, dada sua dificuldade em se transformar realidade. Em dezembro de 2018 estava prevista a universalização da residência médica, com obrigatoriedade da realização de um ano da residência de MFC pela maioria dos egressos ${ }^{13}$. Chegamos sequer perto disso?

Em que pese a superioridade do PMPB como estratégia de provimento e fixação de médicos, não se pode julgar a incapacidade do programa em atender problemas que fogem do escopo de suas ações. 
Diferentes problemas serão abordados com políticas específicas: a fragilidade do acesso está sendo impulsionada com o Programa Saúde na Hora, a qualificação e estruturação da APS será melhorada através do novo modelo de financiamento, o Previne Brasil.

O fortalecimento da residência em MFC será impulsionado com o incentivo municipal de apoio a formação na APS, em consonância com as ações do Previne Brasil. Através dessa ação do Ministério da Saúde, as ESF que são campo de prática de residência em MFC ou área profissional de saúde na APS poderão receber custeio mensal adicional de até $\mathrm{R} \$ 15$ mil. Ao contrário de políticas anteriores ${ }^{14}$, que colocavam o ônus do incentivo adicional às residências apenas sobre estados e municípios, através desse incentivo, o Governo Federal assume a responsabilidade de cofinanciar a formação de profissionais qualificados, estimulando gestores a investir nas residências na APS e valorizando o trabalho em equipe.

Contudo, é importante notar que a residência em MFC é um modelo de formação que não permitirá ampliar a formação de especialistas no país na velocidade em que o SUS necessita. Do lado da oferta, atualmente existem aproximadamente 6 mil especialistas em MFC (muitos não atuando na assistência ou mesmo na especialidade), 3 mil vagas em programas de residência, com dois terços de vagas ociosas. Do lado da demanda, atualmente existem 43 mil ESF. Utilizando a residência como modelo exclusivo de formação de especialistas, levariam décadas até o Brasil atingir o número ideal de MFCs para sua população. Além disso, a residência médica também não resolve o problema da interiorização profissional, uma vez que os programas de residência estão localizados nas médias e grandes cidades. Países muito menores que o Brasil, como Portugal ${ }^{15}$, aperfeiçoaram e titularam os médicos que estavam atuando na APS do país até atingirem o número de especialistas necessários às necessidades dos seus sistemas de saúde. O Brasil segue o mesmo caminho. Com essas diversas ações de valorização da especialidade, o Ministério da Saúde investe nas estratégias de formação de MFCs através da residência e da especialização, buscando formar o contingente de MFC que o Brasil necessita, equilibrando da melhor maneira possível a qualidade da formação com a necessidade da população. Todas as pessoas devem ter acesso ao seu MFC. E as pessoas não podem esperar por décadas para ter esse desejo realizado.

O PMPB será executado pela Agência para o Desenvolvimento da Atenção Primária à Saúde (Adaps). Ao criticar na Adaps o risco de mercantilização da saúde, foi dito ser importante que "o respeito ao ser humano e as necessidades de saúde se sobreponham aos valores e às necessidades do mercado"12, afirmação que está correta. Mas é importante juntar aos interesses do mercado os interesses políticos e ideológicos, das corporações e categorias profissionais, da academia e de antigos gestores, todos igualmente preocupados em defender seus projetos e legados. Os representantes desses interesses buscam também os impor, sob o argumento comum entre todos de ser o melhor para a população. E isso faz parte do processo democrático. Mas boas políticas públicas devem basear-se nas melhores evidências e na capacidade de entrega de serviços de qualidade à população e ser avaliadas pelo que trouxeram de bom e avançar em suas inerentes insuficiências.

AAdaps será um serviço social autônomo que trará grande agilidade e eficiência na operacionalização do PMPB, respeitando os princípios constitucionais da administração pública. As críticas à Adaps carecem de subsídio factual na sua fundamentação, situando-se no campo da especulação. Agestão pública precisa encontrar melhores formas de entregar à população o benefício esperado a partir da coleta de impostos. A Adaps é um exemplo inovador nesse sentido. 
Por fim, matérias de divulgação do PMPB destacaram entre os princípios contemplados nessa política a meritocracia e a equidade, o que causou surpresa naqueles que consideram esses princípios como antagônicos. Mas o PMPB justamente equaciona esses princípios, através da seleção e gratificação profissional baseada em mérito com distribuição desses profissionais de maneira equânime no país. Esse é o desafio de uma boa política pública, equilibrar interesses que parecem antagônicos, com objetivo de atender melhor as pessoas.

\section{Contribuição dos autores}

Redação preliminar: LW. Revisão crítica da versão preliminar: LW, OPD e EH.

Todos os autores aprovaram a versão final e concordaram com prestar contas sobre todos os aspectos do trabalho, sendo LW, OPA e EH os acrônimos do nome dos autores.

\section{Referências}

1. Presidência da República. Lei № 13.958, de 18 de dezembor de 2019. Institui o Programa Médicos pelo Brasil, no âmbito da atenção primária à saúde no Sistema Único de Saúde (SUS), e autoriza o Poder Executivo federal a instituir serviço social autônomo denominado Agência para o Desenvolvimento da Atenção Primária à Saúde (Adaps). Brasília: Diário Oficial da União; 2019.

2. Londoño E. Médicos cubanos reclamam de 'escravidão' em trabalho no Brasil. Folha de S. Paulo. 29 set 2017; Disponível em: https:// www1.folha.uol.com.br/cotidiano/2017/09/1922901-medicos-cubanos-reclamam-de-escravidao-em-trabalho-no-brasil.shtml

3. Cambrivoli F. Na fronteira pelo diploma médico. Estado de S. Paulo. 25 set 2019; Disponível em: https://www.estadao.com.br/infograficos/ cidades, na-fronteira-pelo-diploma-medico, 1028800

4. Scheffer M, et al. Demografia Médica no Brasil 2018. São Paulo: FMUSP, CFM, Cremesp, 2018. 286 p.

5. Instituto Brasileiro de Geogragia e Estatística. Classificação e caracterização dos espaços rurais e urbanos do Brasil: uma primeira aproximação. Rio de Janeiro: IBGE, 2017. 84p.

6. Conselho Nacional de Secretarias Municipais de Saúde. Novo Modelo de Financiamento de Custeio da Atenção Primáriaà Saúde [acesso em 15 dez 2019].Disponível em: https://www.conasems.org.br/wp-content/uploads/2019/11/Conasems_NotaFinanciamentoAB_19nov19-1. pdf

7. Sociedade Brasileira de Medicina de Família e Comunidade. Currículo Baseado em Competências para Medicina de Família e Comunidade. Rio de Janeiro: SBMFC, 2015. Disponível em: http://www.sbmfc.org.br/wp-content/uploads/media/Curriculo\%20Baseado\%20em\%20 Competencias(1).pdf

8. Conselho Nacional de Secretários de Saúde. No Senado Federal, Conass apoia o Programa Médicos pelo Brasil [acesso em 15 dez 2019]. Disponível em: https://www.conass.org.br/no-senado-federal-conass-apoia-o-programa-medicos-pelo-brasil/

9. Associação Médica Brasileira. Médicos pelo Brasil é uma avanço histórico para a saúde [acesso em 15 dez 2019]. Disponível em: https:// amb.org.br/noticias/medicos-pelo-brasil-e-um-avanco-historico-para-a-saude-avalia-amb/

10. Sociedade Brasileira de Medicina de Família e Comunidade. Análise da SBMFC em relação ao Programa Médicos pelo Brasil [acesso em 15 dez 2019]. Disponível em: https://www.sbmfc.org.br/wp-content/uploads/2019/08/análise-em-relação-ao-Programa-Médicospelo-Brasil-proposto-pelo-Ministério-da-Saúde-MS-.pdf

11. Melo Neto AJ, Barreto DS. Programa Médicos pelo Brasil: inovação ou continuidade. Rev Bras Med Fam Comunidade. 2019;14(41):1-9. https://doi.org/10.5712/rbmfc14(41)2162

12. Anderson, MIP. Médicos pelo Brasil e as políticas de saúde para a Estratégia Saúde da Família de 1994 a 2019: caminhos e descaminhos da Atenção Primária no Brasil. Rev Bras Med Fam Comunidade. 2019;14(41):1-16. https://doi.org/10.5712/rbmfc14(41)2180

13. Presidência da República. Lei № 12.871, de 22 de outubro de 2013. Institui o Programa Mais Médicos, altera as Leis no 8.745, de 9 de dezembro de 1993, e № 6.932, de 7 de julho de 1981, e dá outras providências. Brasília: Diário Oficial da União; 2013.

14. Ministério da Saúde . Portaria № 3.147, de 28 de dezembro de 2012. Institui as especificações "preceptor" e "residente” no cadastro do médico que atua em qualquer uma das Equipes de Saúde da Família previstas na Política Nacional de Atenção Básica, de que trata a Portaria nº 2.488/GM/MS, de 21 de outubro de 2011. Brasília: Diário Oficial da União; 2012.

15. Ramos V. O ressurgimento da medicina familiar. Revista Crítica de Ciências Sociais. 1987;23:157-68. 